\title{
Additional effects of fluid on the piston
}

\author{
Daniel Himr ${ }^{\mathrm{a}}$, Vladimír Habán, Jan Kůrečka, and František Pochylý
}

Brno University of Technology, Faculty of Mechanical Engineering, Energy Institute, Victor Kaplan Department of Fluid Engineering, Technická 2, 616 69, Brno, Czech Republic

\begin{abstract}
Additional effect of fluid on the solid body is often ignored phenomenon. Although it is possible to do an analytic derivation of equations describing additional effects for many various conditions, it can be difficult to solve them. The paper shows pressure pulsations in the pipe with piston solved by transfer matrix and harmonic analysis with ANSYS Acoustics, where effect of second viscosity is included.
\end{abstract}

\section{Introduction}

The classic mechanics is based on the law, that all forces acting on the body bring about the acceleration according to (1):

$$
\sum F_{i}=m \cdot \frac{\mathrm{d}^{2} x_{i}}{\mathrm{~d} t^{2}}
$$

The forces can act throughout the volume of a body (e.g. gravity force), on the surface (e.g. pressure force) or at a point. The forces can depend on the acceleration, velocity or position. When only one degree of freedom is supposed (see figure 1), the equation of motion for a rigid body can be written in the following form:

$$
m \cdot \frac{\mathrm{d}^{2} x_{i}}{\mathrm{~d} t^{2}}+b \cdot \frac{\mathrm{d} x_{i}}{\mathrm{~d} t}+k \cdot x_{i}=F_{i},
$$

where $F_{i}$ is an exciting force, damping and stiffness originate from body mounting. The mass represents an effect

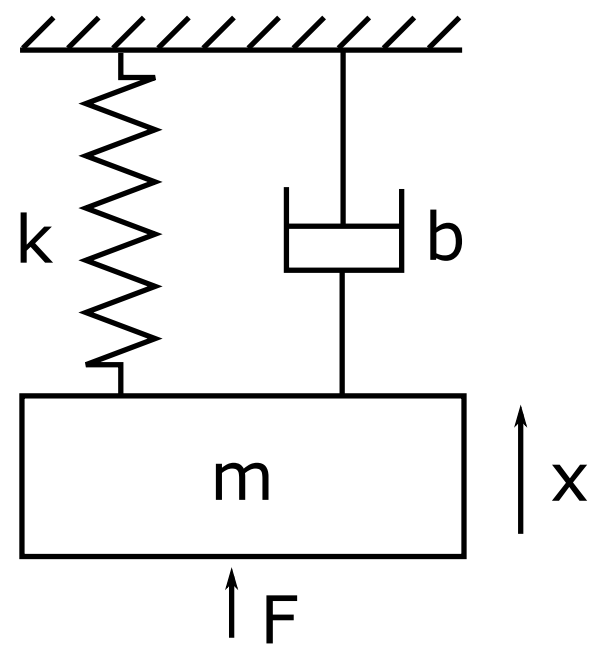

Fig. 1. Body with one degree of freedom

of innertia, the damping represents an energy loss and the stiffness represents a returnable part of the energy, which is connected with a deformation.

\footnotetext{
a e-mail: himr@fme.vutbr.cz
}

Fluids also behave according to (1). Thus, it is possible to apply appropriate assumptions and to derive an equation of motion for fluids (3) [1].

$$
\frac{\partial v_{i}}{\partial t}+\frac{\partial v_{i}}{\partial x_{j}} \cdot v_{j}+\frac{1}{\rho} \cdot \frac{\partial p}{\partial x_{i}}-\frac{1}{\rho} \cdot \frac{\partial \Pi_{i j}}{\partial x_{j}}=g_{i}
$$

Here, an energy loss is represented by the stress tensor $\Pi_{i j}$, which is defined as follows:

$$
\Pi_{i j}=\eta \cdot\left(\frac{\partial v_{i}}{\partial x_{j}}+\frac{\partial v_{j}}{\partial x_{i}}\right)+\delta_{i j} \cdot \eta_{2} \cdot \frac{\partial v_{k}}{\partial x_{k}},
$$

where condition $\eta_{2}=-\frac{2}{3} \cdot \eta$ is often used (e.g. [2]), but this condition originate from Stokes' work [3] and is valid only for monoatomic gases. It means that the gas is always in the thermodynamic equilibrium. This assumption fails in case of polyatomic gases and liquids [4].

When the fluid structure interaction is solved, equations (2) and (3) can be used together with conditions that velocity and stress of the body surface is the same as velocity and stress of the fluid on this surface. Thus, it is possible to derive an equation of motion for a body in the fluid:

$$
\left(m+m_{a}\right) \cdot \frac{\mathrm{d}^{2} x_{i}}{\mathrm{~d} t^{2}}+\left(b+b_{a}\right) \cdot \frac{\mathrm{d} x_{i}}{\mathrm{~d} t}+\left(k+k_{a}\right) \cdot x_{i}=F_{i},
$$

where added mass, added damping and added stiffness originate from the simple fact; when the body is to move, also surrounding fluid has to move. The additional effects can be neglected in some cases. E.g. motion in the air, because the air has low density, but water is almost thousand times thicker. Thus, additional effects start being important.

From practical point of view, eigenfrequency of the body is changed when it is submerged in the water. When this effect is overlooked it can cause problems with vibrations (e.g. runner blades) [5].

Equation (5) is not universal. Its shape can differ and depends on the body geometry, fluid properties, whether the fluid flows or not. More information can be found e.g. in [6]. Thus, equation (5) is simplified version of more complex equation, where non-linear terms are neglected. It causes that the range of validity is limited. 
Table 1. System properties

\begin{tabular}{lll}
\hline Entry & Value & Unit \\
\hline Length & 1 & $\mathrm{~m}$ \\
Cross-section & 1 & $\mathrm{~m}^{2}$ \\
Diameter & $\approx 1.128$ & $\mathrm{~m}$ \\
Fluid density & 1000 & $\mathrm{~kg} \mathrm{~m}^{-3}$ \\
Speed of sound & 1000 & $\mathrm{~m} \mathrm{~s}^{-1}$ \\
Shear viscosity & 0 & $\mathrm{~m}^{2} \mathrm{~s}^{-1}$ \\
\hline
\end{tabular}

\section{Model task}

The problem of fluid additional effects is shown in this paper on a simple example. It is a piston, which is moving in a pipe as shown in figure 2 . The pipe has dead ends and

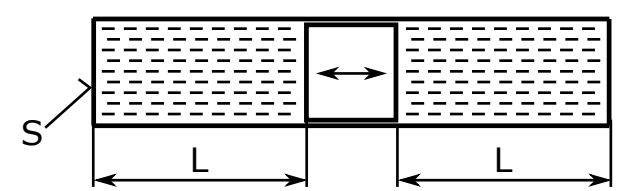

Fig. 2. Solved task; a piston oscillating in the pipe.

rigid walls. The piston oscillates in the axial direction and there is no gap between the piston and the pipe wall. Thus, the fluid cannot flow from the one side of the piston to the other and vice versa. No friction between piston and pipe is assumed and the piston has no mass. The system properties are listed in table 1.

The task is symetrical. Thus, it is possible to solve only one half. The piston velocity is described as follows:

$$
v=i \cdot \frac{\omega}{1000} \cdot \mathrm{e}^{i \cdot \omega \cdot t}
$$

The corresponding displacement of the piston is:

$$
x=\frac{v}{i \cdot \omega} .
$$

Now, Laplace transform is applied to (5). When mass, damping and stiffness are assumed to be zero, it is possible to write:

$$
\left(m_{a} \cdot s^{2}+b_{a} \cdot s+k_{a}\right) \cdot \hat{x}=\hat{F} .
$$

When $i \cdot \omega$ is substituted for the parameter of Laplace transformation, it is possible to write:

$$
\left(k_{a}-m_{a} \cdot \omega^{2}\right)+i \cdot \omega \cdot b_{a}=\frac{\hat{F}}{\hat{x}} .
$$

When frequency amplitude characteristic is known (e.g. from a simulation or an experiment), it is possible to determine added mass and stiffness from real part, which is a parabolic function of the angular speed, and added damping from imaginary part, which is a linerar function of the angular speed.

\subsection{Flow simulation}

Two approaches to solve the fluid flow were used. The first one is by transfer matrix, the other one is with a commercial software ANSYS Acoustics. The solution by transfer matrix of the system originates from the momentum equation (3) and the continuity equation (10):

$$
\rho \cdot a^{2} \cdot \frac{\partial v}{\partial x}+\frac{\partial p}{\partial t}=0 .
$$

When the laminar flow is supposed, it is possible to write the result as follows:

$$
\hat{u}(x, s)=P(x, s) \cdot \hat{u}(0, s),
$$

where $u$ represents a state vector:

$$
\hat{u}(x, s)=\left(\begin{array}{l}
\hat{v}(x, s) \\
\hat{p}(x, s)
\end{array}\right)
$$

and $P$ represents the transfer matrix:

$P=\left(\begin{array}{cc}\cosh \left(\sqrt{\frac{s \cdot B}{A \cdot C}} \cdot x\right) & -\sqrt{\frac{s \cdot A}{B \cdot C}} \cdot \sinh \left(\sqrt{\frac{s \cdot B}{A \cdot C}} \cdot x\right) \\ -\sqrt{\frac{B \cdot C}{s \cdot A}} \cdot \sinh \left(\sqrt{\frac{s \cdot B}{A \cdot C}} \cdot x\right) & \cosh \left(\sqrt{\frac{s \cdot B}{A \cdot C}} \cdot x\right)\end{array}\right)$,

where:

$$
\begin{aligned}
A & =\frac{1}{\rho}+\frac{2 \cdot \eta+\eta_{2}}{\rho^{2}} \cdot \frac{s}{a^{2}}, \\
B & =s-\frac{s \cdot \mathrm{J}_{1}(y)}{s \cdot \mathrm{J}_{1}(y)-\frac{y}{2} \cdot \mathrm{J}_{0}(y)}, \\
C & =a^{2} \cdot \rho, \\
y & =\frac{D}{2} \cdot i \cdot \sqrt{\frac{s \cdot \rho}{\eta}} .
\end{aligned}
$$

Equation (15) represents an influence of velocity profile, which does not have any effect because zero shear viscosity is supposed.

Thus, equation (11) has a solution, when two boundary conditions are given; zero velocity at dead end of the pipe and excitation (6) at the other end. The computed pressure on the piston allows determining the force as a function of the angular velocity. The function has the real and imaginary part and it is possible to determine additional effects, when (9) is used.

The simulation was performed with constant second viscosity and with the second viscosity as a function of angular velocity:

$$
\eta_{2}=\frac{h}{\omega} .
$$

Simulation in ANSYS Acoustics was performed using Harmonic response analysis. Geometry was represented by one-meter-long cylinder with cross section one square meter and this volume was meshed with appropriate element density. Boundary conditions of rigid wall (zero particle velocity) was used on cylindrical surface and on one end, on the opposite end acoustic surface velocity in normal direction with amplitude of $0.001 \mathrm{~m} \mathrm{~s}^{-1}$ was applied. Physical constants were identical to the ones in Table 1 except for shear viscosity which was $10^{-6} \mathrm{~m}^{2} \mathrm{~s}^{-1}$. Unfortunately, when zero shear viscosity is used, the software does not compute effect of second viscosity either. It is unpleasant property of this software, but, in this case, the effect of shear viscosity is small in comparison with the effect of second viscosity.

The ANSYS Acoustics was used because it has the second viscosity as an optional parameter. This software is an extension of ANSYS Mechanical and is designated for simulations of noise propagation, sound acoustics, vibroacoustics etc. The solver is based on the wave equation. 


\section{Results}

This section is divided into three subsections. First one is devoted to comparison of result by the transfer matrix with ANSYS Acoustic result. Then, an influence of the second viscosity on the result was investigated and finally, computation with the frequency depended second viscosity was performed. The range of computed frequencies is from $0 \mathrm{~Hz}$ to $1273 \mathrm{~Hz}\left(0 \mathrm{rad} \mathrm{s}^{-1}\right.$ to $\left.8000 \mathrm{rad} \mathrm{s}^{-1}\right)$.

\subsection{Transfer matrix vs commercial software}

Computation by transfer matrix gives the same results as computation with ANSYS Acoustics. The difference is given only by the step on the solved frequency; see figures 3 and 4.

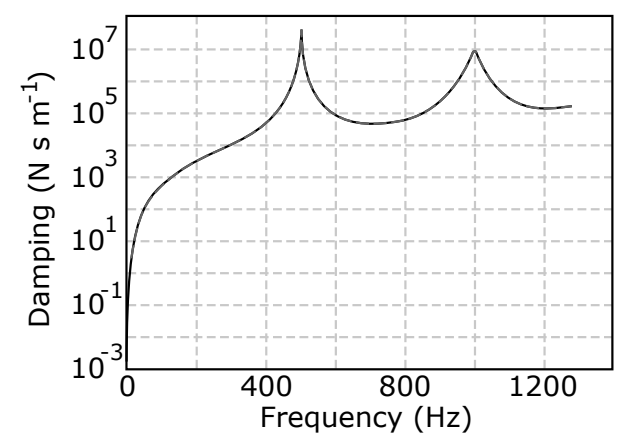

Fig. 3. Comparison of computed damping by transfer matrix (solid black curve) and ANSYS (grey dashed curve)

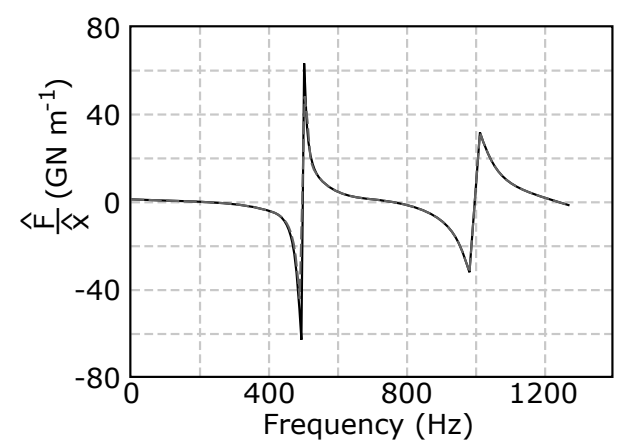

Fig. 4. Comparison of computed real part of the result by transfer matrix (solid black curve) and ANSYS (grey dashed curve)

\subsection{Influence of second viscosity}

Pressure pulsation with variable value of second viscosity gives different damping. The second viscosity is independent on the frequency in this case. The results are compared in the figure 5. Generally, the higher value of second viscosity the higher value of damping, but the damping is more intense for lower values of the second viscosity at the eigenfrequency of the system.

The real part of the result for higher values of the second viscosity is shown in figure 6 . The result for lower values of the second viscosity is shown in figure 7. Unlike the

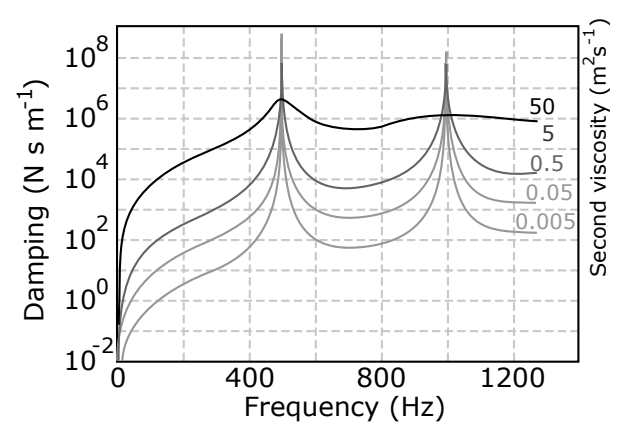

Fig. 5. Evaluated damping for various values of the second viscosity

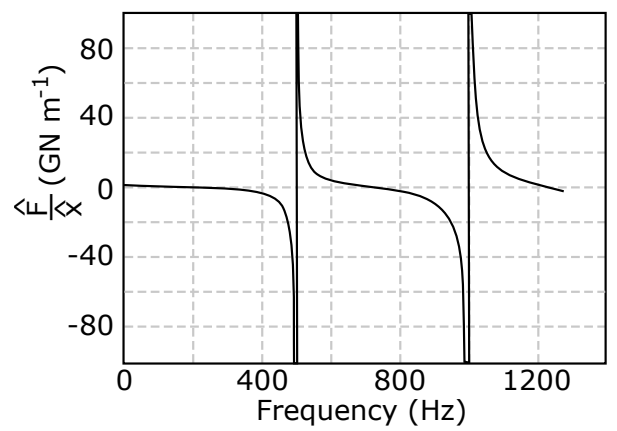

Fig. 6. The real part of the result for values of the second viscosity $\left(50 \mathrm{~m}^{2} \mathrm{~s}^{-1}, 5 \mathrm{~m}^{2} \mathrm{~s}^{-1}\right.$ and $\left.0.5 \mathrm{~m}^{2} \mathrm{~s}^{-1}\right)$

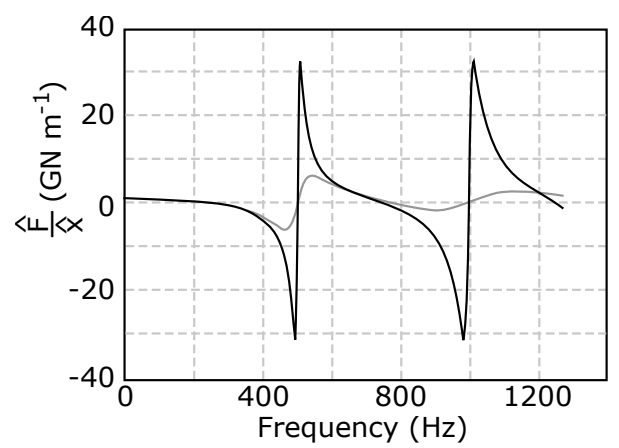

Fig. 7. The real part of the result for values of the second viscosity $0.05 \mathrm{~m}^{2} \mathrm{~s}^{-1}$ (black curve) and $0.005 \mathrm{~m}^{2} \mathrm{~s}^{-1}$ (grey curve)

imaginary part of the result, the real part is almost the same for second viscosities $0.5 \mathrm{~m}^{2} \mathrm{~s}^{-1}, 5 \mathrm{~m}^{2} \mathrm{~s}^{-1}$ and $50 \mathrm{~m}^{2} \mathrm{~s}^{-1}$.

Figures 5 to 7 are drawn according to transfer matrix (or ANSYS, the results are the same). When model according to (5) is used it is possible to get valid result up to frequency $160 \mathrm{~Hz}$. Figure 8 compares result by transfer matrix and (5). Added stiffness is $10 \mathrm{GN} \mathrm{m}^{-1}$ and added mass is $350 \mathrm{~kg}$. The error of description by (5) is drawn in figure 9. One can see that the relative error is small for low frequencies. The error is $1 \%$ for $160 \mathrm{~Hz}$, which is approximately $1 / 3$ of the first eigenfrequency. From this point the error is rapidly growing.

\subsection{Frequency depended second viscosity}

When the second viscosity is assumed to follow (18), the damping is higher for low frequencies and decreases with increasing frequency. One computation was performed with hysteresis damping coefficient $h=5000 \mathrm{~m}^{2} \mathrm{~s}^{-2}$. 
EPJ Web of Conferences

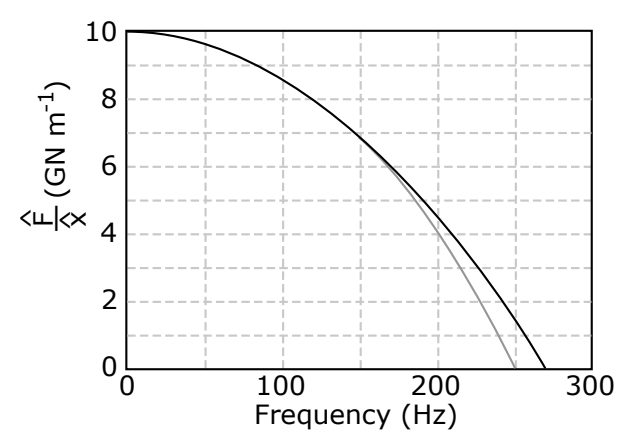

Fig. 8. Real part of the result. Grey curve is obtained by transfer matrix, the black curve is drawn according to (5).

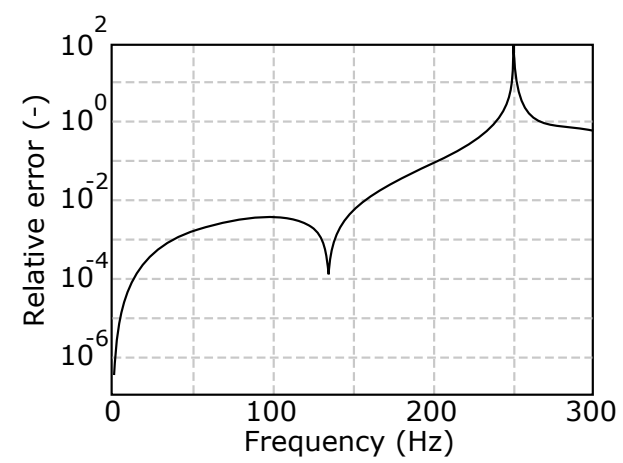

Fig. 9. The error of model with additional effects (5)

Figure 10 compares the result with simulation where constant second viscosity was used. The damping is stronger than case with second viscosity $50 \mathrm{~m}^{2} \mathrm{~s}^{-1}$ but converges to case $5 \mathrm{~m}^{2} \mathrm{~s}^{-1}$ with increasing frequency. The result is the same for angular velocity $1000 \mathrm{rad} \mathrm{s}^{-1}$.

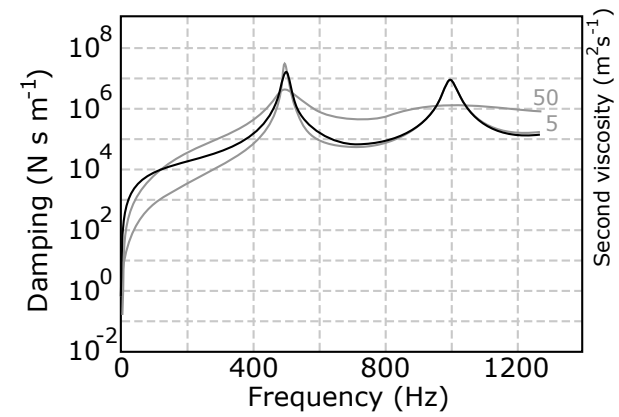

Fig. 10. Black curve shows damping when second viscosity follows hyperbolic function(18). The result is compared with cases where constant second viscosity is used (grey curves).

The real part of the result does not exhibit such a big deviations from the case with constant second viscosity; see figure 11.

\section{Conclusion}

The paper shows possibilities how to determine additional effects of a fluid on a piston. The solution was performed by transfer matrix and with ANSYS Acoustics software. The comparison showed that both approaches give the same results. The complicated models of the fluid flow can be replaced with the momentum equation of rigid body with

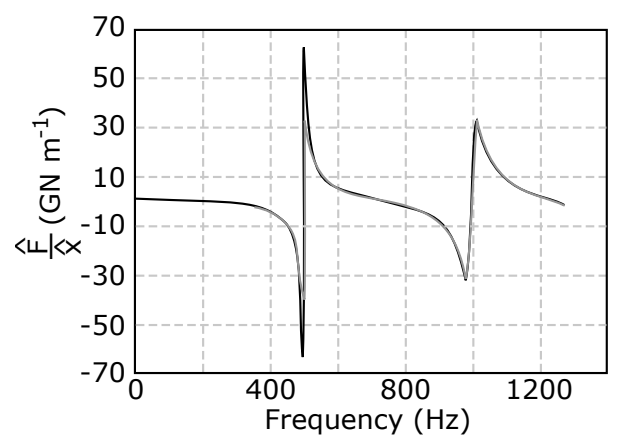

Fig. 11. Real part of the result when second viscosity follows the hyperbolic function (black curve) is compared with the case with second viscosity is $5 \mathrm{~m}^{2} \mathrm{~s}^{-1}$ (grey curve)

added mass, added damping and added stiffness to describe the force acting on the piston. The added mass and added stiffness are constant at low frequencies (up to $1 / 3$ the of first eigenfrequency). The added damping is a linear function of the angular velocity at low frequencies, when the second viscosity follows (18). When the second viscosity is constant the added damping is a parabolic function.

The attention was also paid to influence of the second viscosity, which has dominant effect on the energy loss in the solved task.

\section{Acknowledgement}

This work is an output of cooperation between projects of Technology Agency of the Czech Republic No. TE02000232 "Special rotary machine engineering centre" and NETME Centre, regional R\&D centre built with the financial support from the Operational Programme Research and Development for Innovations within the project NETME Centre (New Technologies for Mechanical Engineering), Reg. no. CZ.1.05/2.1.00/ 01.0002 and, in the follow-up sustainability stage, supported through NETME CENTRE PLUS (LO1202) by financial means from the Ministry of Education, Youth and Sports under the "National Sustainability Programme I."

\section{Nomenclature}

$\begin{array}{lll}a & \left(\mathrm{~m} \mathrm{~s}^{-1}\right) & \text { speed of sound, } \\ b & \left(\mathrm{~N} \mathrm{~s} \mathrm{~m}^{-1}\right) & \text { damping, } \\ b_{a} & \left(\mathrm{~N} \mathrm{~s} \mathrm{~m}^{-1}\right) & \text { added damping, } \\ D & (\mathrm{~m}) & \text { diameter, } \\ F & (\mathrm{~N}) & \text { force, } \\ g & \left(\mathrm{~m} \mathrm{~s}^{-2}\right) & \text { gravity acceleration, } \\ h & \left(\mathrm{~m}^{2} \mathrm{~s}^{-2}\right) & \text { hysteresis damping coefficient, } \\ k & \left(\mathrm{~N} \mathrm{~m}^{-1}\right) & \text { stiffness, } \\ k_{a} & \left(\mathrm{~N} \mathrm{~m}^{-1}\right) & \text { added stiffness, } \\ L & (\mathrm{~m}) & \text { length, } \\ m & (\mathrm{~kg}) & \text { mass, } \\ m_{a} & (\mathrm{~kg}) & \text { added mass, } \\ P & (-) & \text { transfermatrix, } \\ p & (\mathrm{~Pa}) & \text { pressure, } \\ S & \left(\mathrm{~m}^{2}\right) & \text { cross-section, } \\ s & \left(\mathrm{~s}^{-1}\right) & \text { parameter of the Laplace transform, } \\ t & (\mathrm{~s}) & \text { time, }\end{array}$




$\begin{array}{lll}u & & \text { state vector, } \\ v & \left(\mathrm{~m} \mathrm{~s}^{-1}\right) & \text { velocity, } \\ x & (\mathrm{~m}) & \text { space coordinates, } \\ \delta_{i j} & (-) & \text { Kronecker delta, } \\ \eta & (\mathrm{Pa} \mathrm{s}) & \text { shear viscosity, } \\ \eta_{2} & (\mathrm{~Pa} \mathrm{~s}) & \text { second viscosity, } \\ \Pi & (\mathrm{Pa}) & \text { stress tensor, } \\ \rho & \left(\mathrm{kg} \mathrm{m}^{-3}\right) & \text { density, } \\ \omega & \left(\mathrm{rad} \mathrm{s}^{-1}\right) & \text { angular velocity, } \\ & & \text { variable after the Laplace transform. }\end{array}$

\section{References}

1. V. L. Streeter, E. B. Wylie, K. W. Bedford, Fluid Mechanics, Ninth Edition (WCB/McGraw Hill, 1998).

2. L. Hružík, M. Vašina, A. Bureček, Advances in Mechanism Design II 44, 137 - 143, (2017).

3. G. G. Stokes, Trans. of the Cambridge phil. soc. 8, 287 $-305,(1845)$.

4. L. Tisza, Physical Review 61, (1942).

5. F. Pochylý, S. Fialová, M. Kozubková, M. Bojko, IOP Conf. Series-Earth and Environmental Science 15, 072027, (2013).

6. C. Conca, A. Osses, J. Planchard, Comp. methods in appl. mech. and eng. 146 (1997) 387 - 405. 